\title{
The peptidyl-prolyl isomerase Pin1 determines parathyroid hormone mRNA levels and stability in rat models of secondary hyperparathyroidism
}

\author{
Morris Nechama, ${ }^{1}$ Takafumi Uchida, ${ }^{2}$ Irit Mor Yosef-Levi,, ${ }^{1}$ Justin Silver, ${ }^{1}$ and Tally Naveh-Many ${ }^{1}$ \\ ${ }^{1}$ Minerva Center for Calcium and Bone Metabolism, Nephrology Services, Hadassah Hebrew University Medical Center, \\ Jerusalem, Israel. ${ }^{2}$ Molecular Enzymology, Tohoku University, Sendai, Japan.
}

\begin{abstract}
Secondary hyperparathyroidism is a major complication of chronic kidney disease (CKD). In experimental models of secondary hyperparathyroidism induced by hypocalcemia or CKD, parathyroid hormone $(P T H)$ mRNA levels increase due to increased PTH mRNA stability. K-homology splicing regulator protein (KSRP) decreases the stability of $P T H$ mRNA upon binding a cis-acting element in the PTH mRNA 3' UTR region. As the peptidyl-prolyl isomerase (PPIase) Pin1 has recently been shown to regulate the turnover of multiple cytokine mRNAs, we investigated the role of Pin1 in regulating PTH mRNA stability in rat parathyroids and transfected cells. The data generated were consistent with Pin1 being a PTH mRNA destabilizing protein. Initial analysis indicated that Pin1 activity was decreased in parathyroid protein extracts from both hypocalcemic and CKD rats and that pharmacologic inhibition of Pin1 increased PTH mRNA levels posttranscriptionally in rat parathyroid and in transfected cells. Pin1 mediated its effects via interaction with KSRP, which led to KSRP dephosphorylation and activation. In the rat parathyroid, Pin1 inhibition decreased KSRP-PTH mRNA interactions, increasing PTH mRNA levels. Furthermore, $P$ in $1^{-/-}$mice displayed increased serum PTH and PTH mRNA levels, suggesting that Pin1 determines basal PTH expression in vivo. These results demonstrate that Pin1 is a key mediator of PTH mRNA stability and indicate a role for Pin1 in the pathogenesis of secondary hyperparathyroidism in individuals with CKD.
\end{abstract}

\section{Introduction}

Parathyroid hormone (PTH) regulates serum calcium and phosphate levels and bone strength. In turn, PTH gene expression, PTH secretion, and parathyroid cell proliferation are determined by serum calcium and phosphate. Dietary calcium depletion and experimental chronic kidney disease (CKD) lead to secondary hyperparathyroidism characterized by increased PTH mRNA levels, serum PTH, and parathyroid cell proliferation (1). The changes in PTH mRNA levels due to calcium depletion as well as CKD are posttranscriptional, affecting mRNA stability $(2,3)$. They are mediated by the regulated binding of trans-acting factors to a 63-nt-long cis-acting AU-rich element (ARE) located in the PTH mRNA 3' UTR $(4,5)$. A 26-nt element within the 63-nt sequence is the minimal protein-binding region and is conserved among species (4). A number of ARE-binding proteins have been identified. Among these, K-homology splicing regulator protein (KSRP) is an example of a decay-promoting factor that recruits the multiprotein $3^{\prime}-5^{\prime}$ exoribonuclease complex, exosome (6), to target mRNAs (7). AU-rich binding factor 1 (AUF1) promotes either decay or stabilization, depending on the mRNA and cell type $(8,9)$. We have previously shown that AUF1 and KSRP have opposite effects on PTH mRNA stability. AUF1 binds to the PTH mRNA 63-nt ARE, increasing PTH mRNA $t_{1 / 2}$ (10). KSRP interacts with the same 63-nt ARE element, but decreases PTH mRNA $t_{1 / 2}$ (11). PTH mRNA interactions with AUF1 and KSRP

Conflict of interest: The authors have declared that no conflict of interest exists. Citation for this article: J. Clin. Invest. 119:3102-3114 (2009). doi:10.1172/JCI39522. are regulated by changes in serum calcium and phosphate concentrations and by CKD $(11,12)$.

The peptidyl-prolyl cis-trans isomerase Pin 1 specifically binds phosphorylated Ser/Thr-Pro protein motifs and catalyzes the cis-trans isomerization of the peptide bonds, thereby changing the biological activity, phosphorylation, and turnover of its target proteins $(13,14)$. Pin 1 consists of an aminoterminal proteinprotein interaction domain, the WW domain, which is involved in Pin 1 binding to its Ser/Thr-Pro target phosphoproteins and a carboxyterminal peptidyl-prolyl cis-trans isomerase (PPIase) domain (15). Pin 1 is the only mammalian enzyme known to specifically catalyze the cis-trans isomerization of Ser-Pro or Thr-Pro peptide bonds $(16,17)$. Pin1-catalyzed conformational regulation has a profound impact on many key proteins involved in various cell functions $(18,19)$. Interestingly, Pin 1 was recently shown to regulate the turnover of ARE-containing mRNAs, mainly cytokine mRNAs, through the interaction and isomerization of ARE-binding proteins. Pin 1 interacts with AUF1 and thereby stabilizes both GM-CSF and TGF- $\beta$ mRNAs $(20,21)$. In contrast to its degrading effect on these mRNAs, AUF1 is a PTH mRNA-stabilizing protein (10). We hypothesized that Pin1 may be involved in the regulation of $P T H$ gene expression.

Here, we identify Pin 1 as a PTH mRNA-regulating protein. We show that Pin 1 activity is decreased in parathyroid extracts from calcium-depleted rats or in CKD rats, in which PTH mRNA levels and stability are increased. Accordingly, Pin 1 inhibition by juglone increases serum PTH and PTH mRNA levels in the rat. This increase in PTH gene expression is posttranscriptional. In transfected cells, 
A
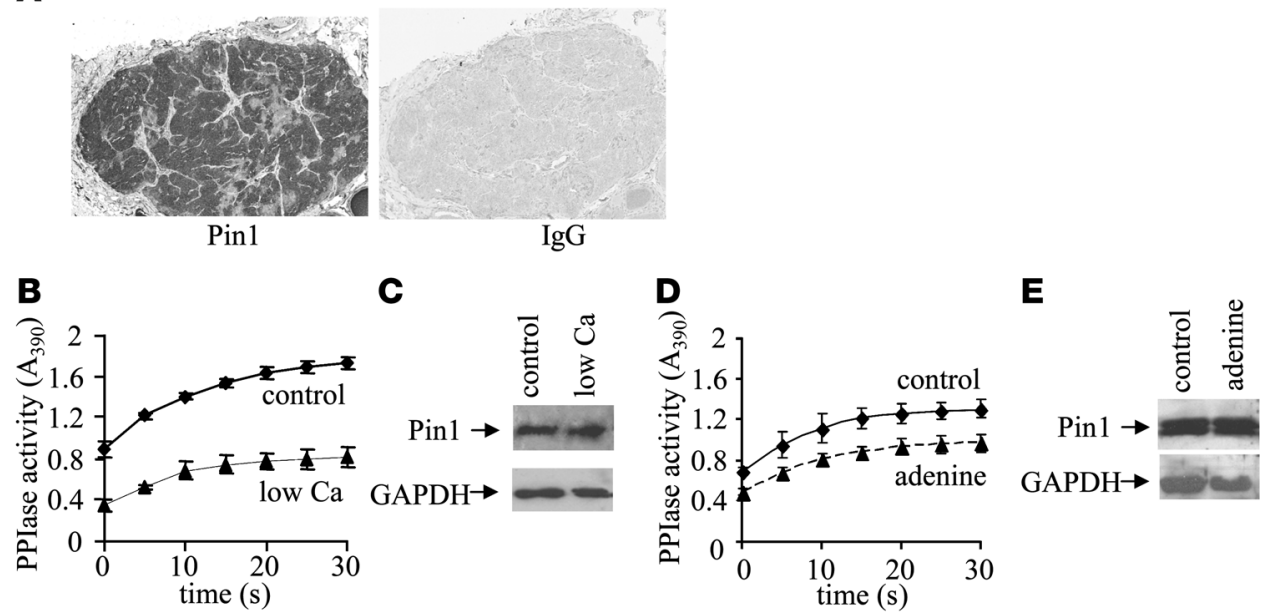

Figure 1

PPlase activity is decreased in parathyroid extracts from rat with secondary hyperparathyroidism due to a calcium-depleted diet or an adenine and high-phosphorus diet. (A) Immunohistochemistry using an anti-Pin1 antibody or IgG showing specific expression of Pin1 in the parathyroid cells and not in interstitial tissue or blood vessels. Original magnification, $\times 200$. (B and D) Pin1 isomerase assays. PPlase activity of parathyroid cytoplasmic lysates from a pool of 5 rats fed a calcium-depleted diet (B) or an adenine and high-phosphorus diet (D) compared with parathyroid extracts from control rats. The results are representative of 3 independent experiments and are presented as mean \pm SEM of 3 measurements from 1 of the experiments (B) and as mean \pm SEM of 3 measurements from the 3 experiments combined (D). The decrease in Pin1 activity in $\mathbf{B}$ and $\mathbf{D}$ was significant $(P<0.05)$ compared with the respective controls at all the time intervals studied. Control: low $\mathrm{Ca}$ or adenine, $P<0.05$ at all time intervals. (C and E) Immunoblot analysis using anti-Pin1 or anti-GAPDH antibodies for parathyroid extracts, as in $\mathbf{B}$ and $\mathbf{D}$.

overexpression of Pin 1 decreases and Pin 1 knockdown increases cotransfected $P T H$ mRNA levels. These effects of Pin 1 are dependent upon the PTH mRNA 3' UTR ARE. Pin1 interacts with KSRP, and Pin1 overexpression leads to KSRP dephosphorylation, which determines KSRP-mediated PTH mRNA decay. In vivo, in the rat, Pin1 inhibition prevents KSRP-PTH mRNA interaction. Finally, we show that $P$ in $1^{-/-}$mice have increased serum PTH and PTH mRNA levels. Our results demonstrate a role for Pin 1 in the regulation of PTH mRNA stability and in the response to calcium depletion and CKD.

\section{Results}

Pin1 activity is decreased in parathyroid extracts of rats with secondary hyperparathyroidism. We have previously reported that dietary calcium depletion and experimental CKD lead to secondary hyperparathyroidism characterized by a posttranscriptional increase in PTH mRNA levels (22). To determine whether the prolyl isomerase Pin 1 is involved in this regulation, we first showed Pin 1 expression in parathyroid tissue by immunohistochemistry of rat parathyroid tissue (Figure 1A). We then analyzed Pin 1 enzymatic activity in parathyroid extracts from control and secondary hyperparathyroidism rats. Rats were fed either a control diet or diets that were calcium restricted or supplemented with adenine and high phosphorus to induce CKD. Both diets increase serum PTH and PTH mRNA levels compared with a control diet fed to rats, with the effect of calcium depletion being greater than that of the adenine diet (11). At the end of the diet period, Pin 1 activity was measured in microdissected parathyroid cytosolic lysates by an isomerase assay (23). PPIase activity was markedly reduced in parathyroid cytoplasmic lysates of both rats fed a calcium-restricted diet and CKD rats compared with parathyroid extracts from control rats (Figure 1, B and D). There was no change in Pin 1 protein levels (Figure 1, C and E). These results indicate that Pin 1 activity is decreased in response to changes in serum calcium and CKD. The decrease in Pin 1 activity inversely correlates with the increased PTH mRNA levels and stability in these models.

Pin1 inbibition by juglone increases PTH $m R N A$ in vivo. The decreased Pin 1 activity in parathyroid extracts from secondary hyperparathyroidism rats suggests that Pin 1 inhibition would lead to increased PTH mRNA levels. We therefore studied the effect of a specific and irreversible Pin1 inhibitor, juglone (23), on PTH gene expression in vivo. Juglone covalently inactivates Pin 1 and accelerates its catabolism by the proteasome (20). Juglone was administered systemically by 2 daily i.p. injections. One hour after the second injection, there was the expected decrease in PPIase activity in the parathyroid cytoplasmic lysates (Figure 2A) and an increase in serum PTH levels (Figure 2B). To study the direct effect of Pin 1 inhibition on PTH expression, we applied juglone topically on the parathyroid glands using a method that we have recently developed to study the effect of direct application of a compound to the parathyroid while maintaining the glands in their physiological milieu (24). After 2 hours of juglone or vehicle administration, treated parathyroid glands were removed and analyzed for PPIase activity, Pin 1 protein levels, and PTH mRNA levels. Pin1 protein levels and PPIase activity were markedly decreased in the juglone-treated parathyroid cytoplasmic lysates (Figure 2, C and D). Importantly, juglone administration markedly increased PTH mRNA levels by both Northern blots and real-time quantitative RT-PCR (qRT-PCR) (Figure 2, E and F). Therefore, Pin1 inhibition by juglone increases $P T H$ gene expression in vivo.

Pin1 inbibition increases PTH $m R N A$ levels by a posttranscriptional mechanism. To study the mechanism of the effect of Pin 1 inhibition by juglone on $P T H$ gene expression, we performed nuclear run-on assays with isolated nuclei from parathyroid glands of rats treated topically with juglone or control rats, as above (Figure 2, C-F). PTH transcription was the same in treated and nontreated parathyroid 
A

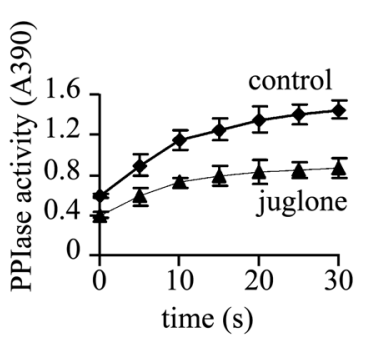

B

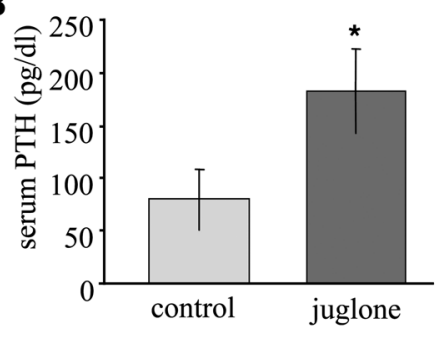

C

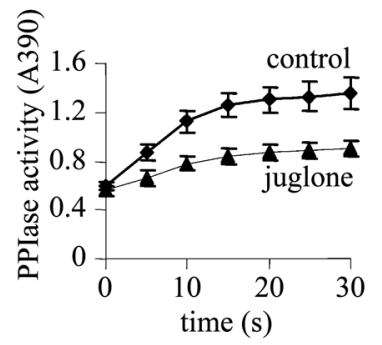

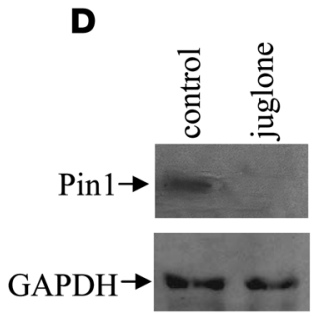

H

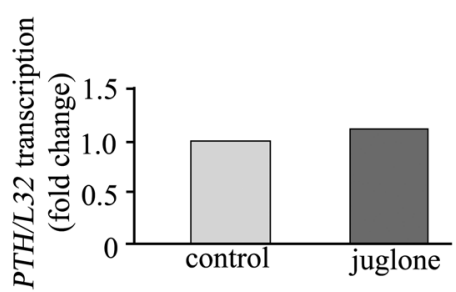

E

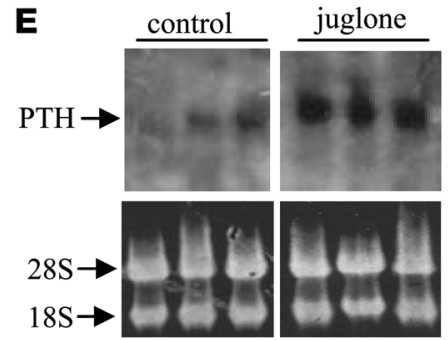

F

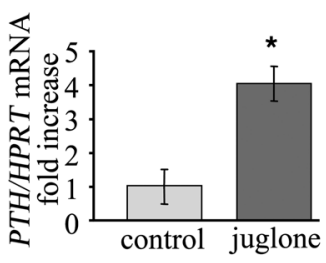

G

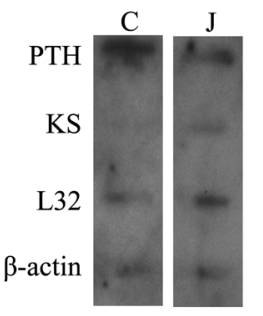

I

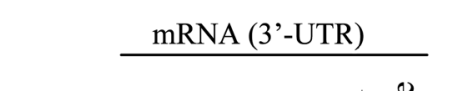

pre-PTH mRNA (intron)

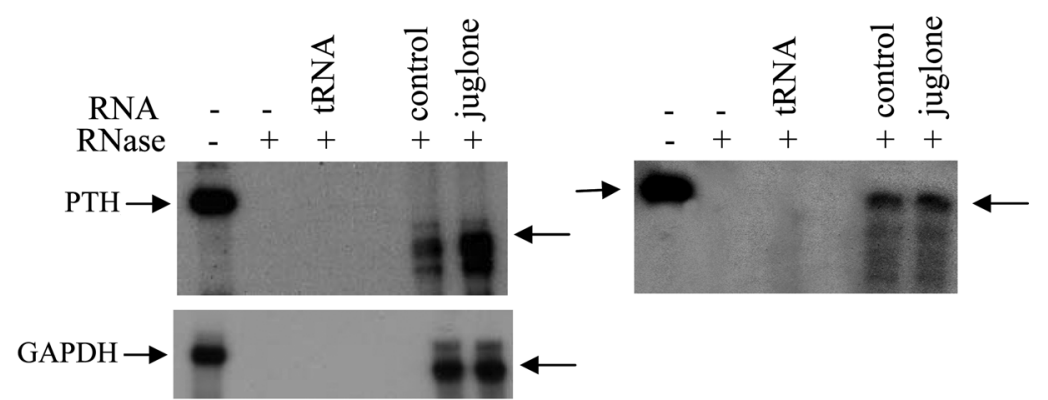

\section{Figure 2}

Pin1 inhibition by juglone increases PTH mRNA in vivo posttranscriptionally. (A and B) Juglone i.p. (A) PPlase activity assays of parathyroid cytoplasmic lysates from rats injected i.p. with juglone or vehicle. (B) Serum PTH in rats as in A. (C-F) Juglone applied topically. (C) PPlase activity of parathyroid cytoplasmic lysates from rats treated with juglone or vehicle applied topically; data in $\mathbf{A}$ and $\mathbf{C}$ are presented as mean \pm SEM $(n=3)$ from 1 of 3 repeat experiments; $P<0.05$ at 5-30 seconds. (D) Immunoblots of the extracts in C for Pin1 and GAPDH. (E) Northern blots of PTH mRNA levels after juglone, in rats as in C. The lanes were run on the same gel but were noncontiguous (white line). (F) qRT-PCR analysis for $P$ TH mRNA in rat parathyroids as in $\mathbf{C}$; ${ }^{*} P<0.05$. (G) Nuclear run-ons of PTH transcription in isolated nuclei of parathyroids from 5 rats in each group after topical juglone $(\mathrm{J})$ or vehicle $(\mathrm{C})$. (H) Quantification of $P T H$ mRNA transcription in $\mathbf{G}$, showing no difference in PTH transcription. The same result was obtained in a repeat experiment. (I) RNase protection analysis (RPA) of total parathyroid RNA from rats after topical juglone or vehicle. Left panels: RPA using an antisense probe for the PTH mRNA 3' UTR or for control GAPDH mRNA. Right panel: RPA using an antisense probe for the first intron (newly transcribed pre-PTH mRNA). The intact probes before and after RNase treatment (arrows to the left and right of the gels, respectively) and hybridization with tRNA as a nonspecific control are shown. Juglone increased $P T H$ mRNA but not the pre-PTH mRNA or GAPDH mRNA.

glands, indicating that the increase in PTH mRNA after Pin 1 inhibition by juglone is posttranscriptional (Figure 2, G and H). To further demonstrate the posttranscriptional effect of juglone on PTH gene expression, we performed RNase protection assays using antisense probes for the PTH mRNA 3' UTR (exon) or for the first intron of PTH mRNA as a marker of newly transcribed pre-PTH mRNA. As shown in Figure 2I, juglone-treated parathyroid glands had higher levels of PTH mRNA compared with control parathyroids based on the RNase protection assay using the PTH mRNA 3' UTR probe, similar to the results obtained by Northern blots and qRT-PCR (Figure 2, E and F). A probe for GAPDH mRNA 3' UTR was used as a control that was not affected by juglone (Figure 2I). In contrast, there was no difference in the pre-mRNA levels for the PTH transcript in juglone-treated parathyroid glands compared with controls, as indicated using the probe for the first intron (Figure 2I). Together, these results indicate that the increased PTH mRNA levels after Pin 1 inhibition by juglone is posttranscriptional, similar to the regulation of PTH gene expression in secondary hyperparathyroidism in which Pin activity is decreased (Figure $1, B$ and D). Thus, the physiological function of Pin 1 may be to decrease $P T H$ gene expression posttranscriptionally.

Pin1 regulates $P T H \mathrm{mRNA}$ stability in cotransfected HEK293 cells, and this is mediated by the PTH mRNA 3' UTR ARE. Since a parathyroid cell line is not available, the direct effect of Pin 1 on PTH mRNA levels was studied in transiently transfected HEK293 cells. Pin1 overexpression decreased PTH mRNA levels with no effect on endogenous L32 or control cotransfected growth hormone (GH) mRNA levels (Figure 3, A-C). The effect of Pin 1 overexpression on 
A

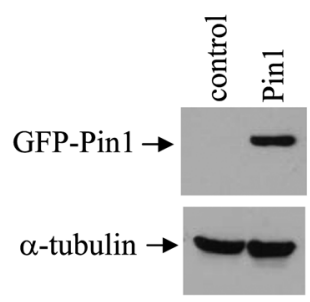

D

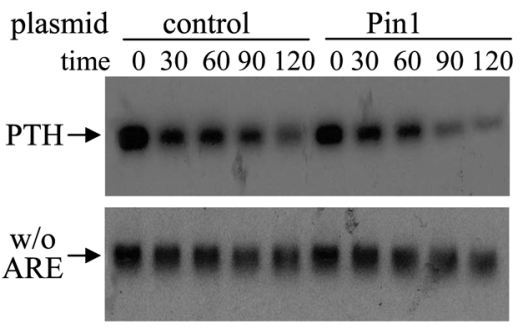

$\mathbf{F}$

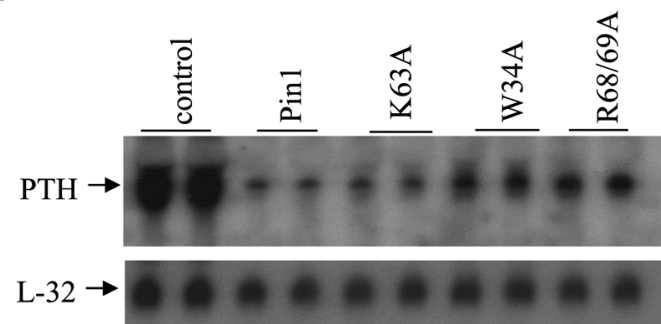

H

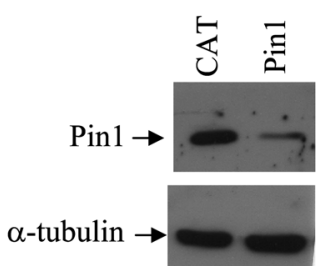

Pin1/ $\alpha$-tubulin (\%) $100 \quad 23.5$

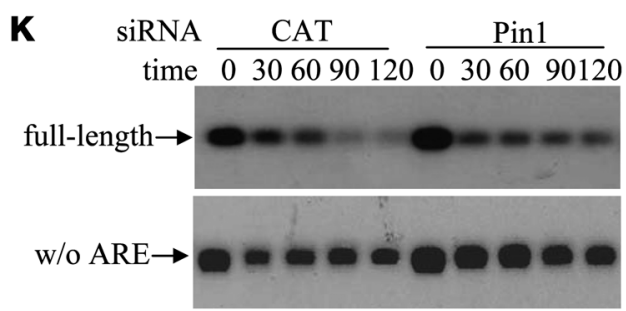

B

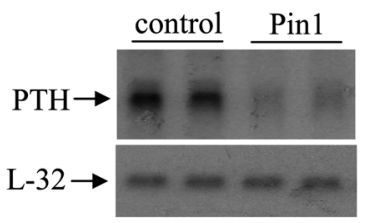

E
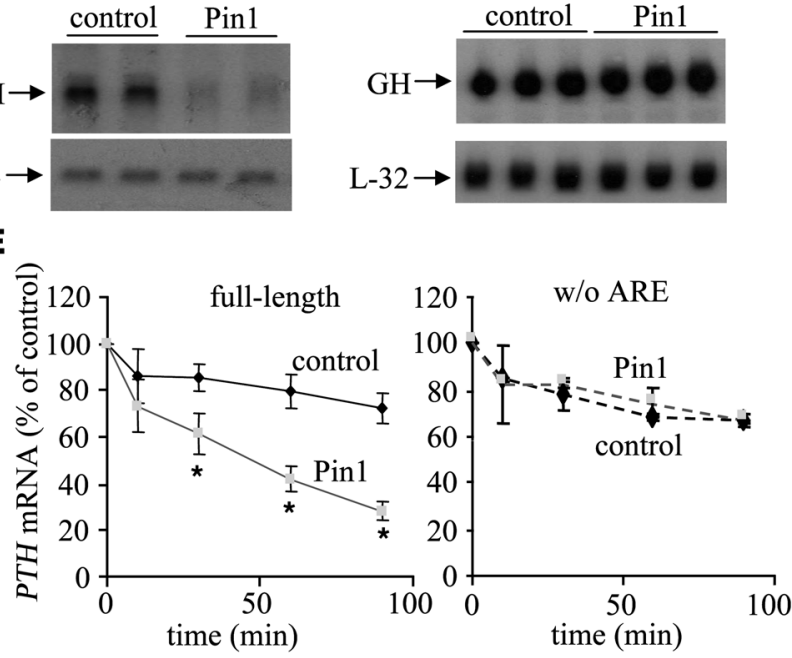

G

I

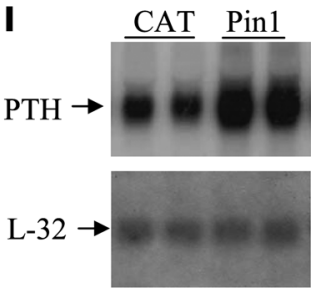

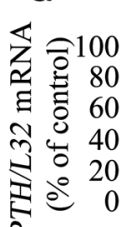

2

L

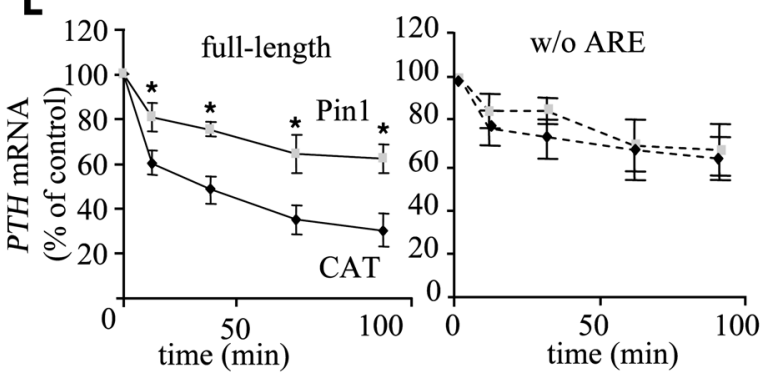

Figure 3

Pin1 regulates PTH mRNA steady-state levels and stability in transfected cells. (A-F) Pin1 overexpression. HEK293 cells were transiently cotransfected with expression plasmids for human PTH or GH and for GFP-Pin1 or control pcDNA3 plasmids. (A) Immunoblots of protein extracts at 48 hours for Pin1 and $\alpha$-tubulin. (B) Northern blots for PTH mRNA. (C) Northern blots for GH mRNA. (D) IVDA for polyadenylated full-length rat PTH mRNA (top) or polyadenylated PTH mRNA without the ARE (bottom) and extracts from cells transfected with GFP-Pin1 or control plasmids. (E) Quantification of the IVDAs in $\mathbf{D}$ and 2 additional IVDAs; mean \pm SEM of intact transcript; ${ }^{*} P<0.05$. (F and $\left.\mathbf{G}\right)$ Cells were transiently cotransfected with expression plasmids for PTH and GFP-Pin1 or Pin1 mutants at the WW domain (W34A) or at the PPlase domain (K63A,R68/69A). (F) Northern blots for PTH mRNA. (G) Quantification of 3 Northern blots; mean $\pm \mathrm{SEM}$; ${ }^{*} P<0.05$. (H-L) Pin1 knockdown. Cells were cotransfected with a human PTH plasmid and either Pin1 or control CAT siRNAs. (H) Immunoblots of Pin1 and $\alpha$-tubulin after transfection. (I) Northern blots for PTH mRNA. (J) Quantification by qRT-PCR analysis for PTH mRNA after Pin1 siRNA. (K) IVDA for full-length rat PTH mRNA (top) or rat PTH mRNA without the ARE (bottom) and extracts from cells with either CAT or Pin1 siRNAs. (L) Quantification of 3 IVDAs; mean \pm SEM of intact transcript at time $0 ;{ }^{*} P<0.05$. 
A
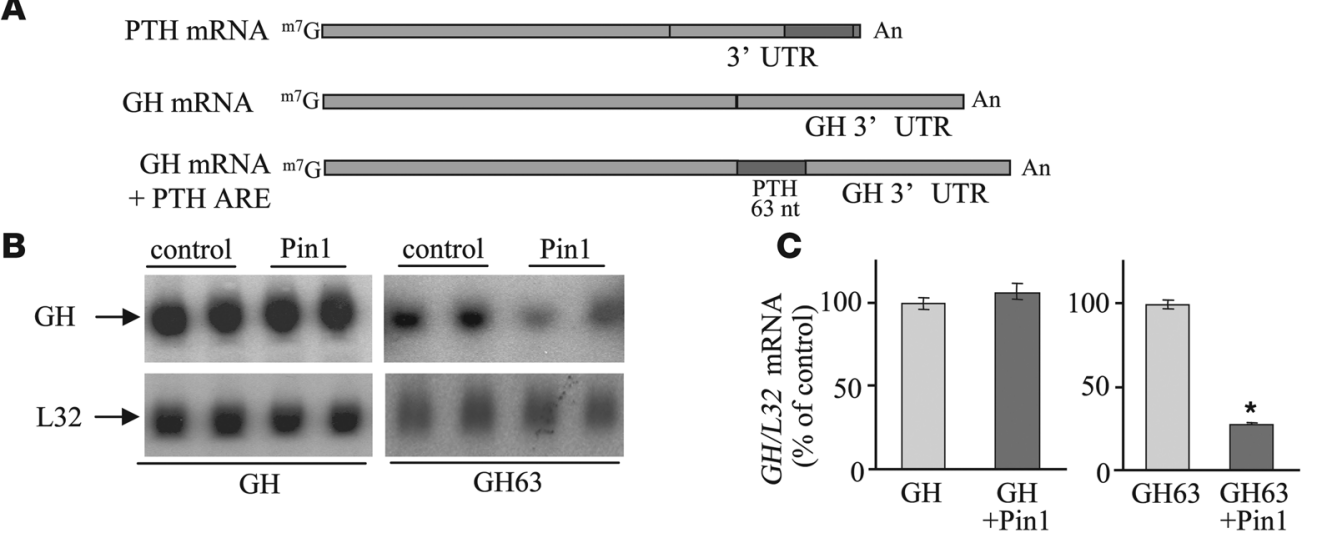

D

E
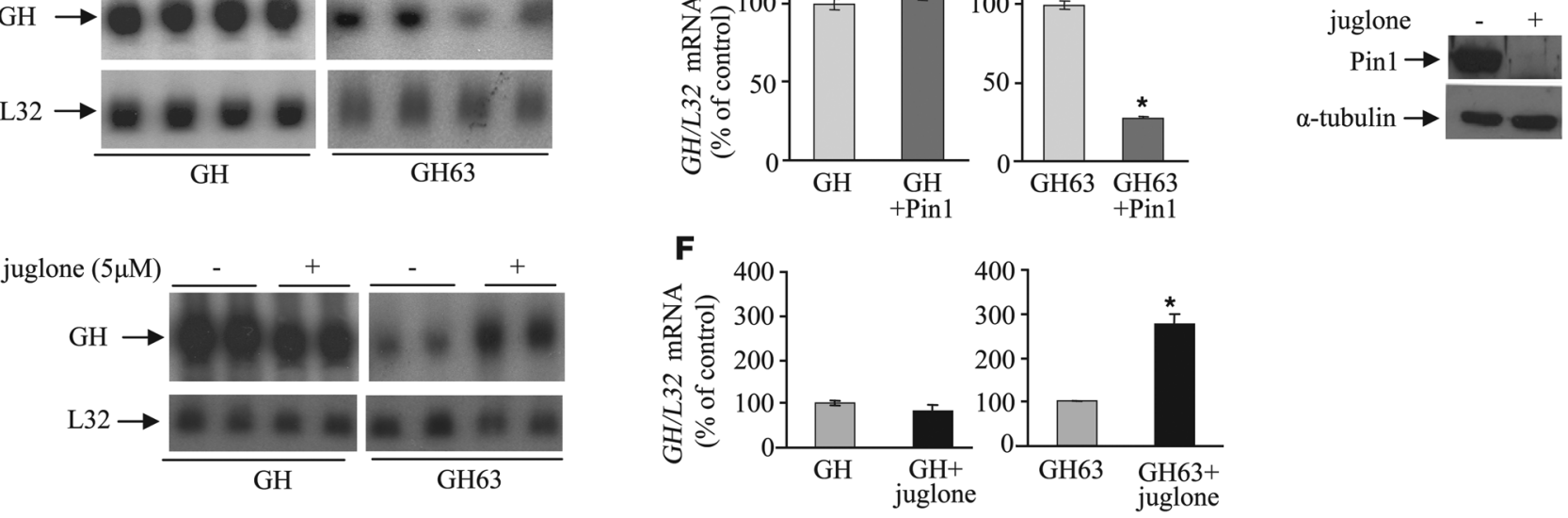

Figure 4

Pin1 regulates $P T H$ mRNA levels through the $P T H$ mRNA ARE. (A) Schematic representation of mRNAs for $P T H$, $G H$, and chimeric reporter $G H$ containing the PTH mRNA ARE (GH63) used for transfections. (B and C) Pin1 overexpression. HEK293 cells were transiently cotransfected with expression plasmids for either GH or a chimeric GH63 (GH63) and GFP-Pin1 or pcDNA3 (control). (B) Northern blot analysis for PTH mRNA. (C) Quantification of 2 Northern blots as in B for GH (left) and GH63 (right); mean \pm SEM; * $P<0.05$ compared with GH63 without Pin1. (D-F) Effect of juglone on GH63. Cells were transiently transfected with expression plasmids for either GH or GH63 and incubated with juglone or vehicle $(-)$. (D) Immunoblots for Pin1 in cell extracts after juglone or vehicle. (E) Northern blots for GH mRNA. (F) Quantification of Northern blots of 3 independent experiments as in $\mathbf{E}\left({ }^{\star} P<0.05\right)$. The lanes in $\mathbf{B}$ and $\mathbf{E}$ were run on the same gel but were noncontiguous (white lines).

PTH mRNA stability was measured by in vitro degradation assays (IVDAs) using transfected cell extracts and polyadenylated fulllength radio-labeled $P T H$ mRNA transcripts (11). Extracts from Pin1-overexpressing cells led to accelerated $P T H$ mRNA decay in vitro compared with extracts from control transfected cell extracts (Figure 3, D and E). The $t_{1 / 2}$ of PTH mRNA decreases from more than 100 minutes with the control transfected extracts to $50 \mathrm{~min}$ utes by the Pin 1 -overexpressing extracts. Pin 1 overexpression, however, had no effect on the in vitro decay of a truncated polyadenylated PTH mRNA transcript lacking the PTH mRNA 3' UTR ARE (Figure 3, D and E), suggesting an ARE-dependent effect of Pin1 to increase PTH mRNA decay.

Both the binding (WW) and isomerase (PPIase) activities of Pin1 are normally required for Pin 1 to regulate the function of its substrates $(16,25)$. To determine whether one or both of these activities are required for the decrease in $P T H$ mRNA levels by Pin1, we cotransfected the PTH expression plasmid with expression plasmids for mutated Pin1 at either the binding domain (W34A) or the PPIase domain (R68/69A, K63A) (19). Pin1 overexpression decreased PTH mRNA levels as before (Figure 3B), as did the K63A mutant (Figure $3, \mathrm{~F}$ and $\mathrm{G}$ ). However, mutants for both the binding domain (W34A) and another PPIase domain (R68/69A) attenuated the downregulation of PTH mRNA induced by WT Pin 1 (Figure 3, F and G). Therefore, Pin 1 overexpression decreases $P T H$ mRNA levels, and both the WW and PPIase domains are necessary for this effect.

Next, we decreased Pin 1 protein levels using siRNAs and assessed the resulting effects on cotransfected PTH mRNA levels. A 75\% reduction in Pin 1 protein levels (Figure $3 \mathrm{H}$ ) led to a 3-fold increase in PTH mRNA levels by both Northern blot and qRT-PCR (Figure 3 , I and J). Similarly, PTH mRNA $t_{1 / 2}$ was prolonged in IVDAs with extracts from Pin1-depleted cells compared with extracts from cells transfected with a control bacterial chloramphenicol acetyltransferase (CAT) siRNA (Figure $3, \mathrm{~K}$ and $\mathrm{L}$ ). The $t_{1 / 2}$ of PTH mRNA increased from 50 minutes by control extracts to more than 100 minutes by the Pin 1 knockdown extracts. As with Pin 1 overexpression (Figure 3, D and E), the $t_{1 / 2}$ of a PTH transcript lacking the $3^{\prime}$ UTR ARE was not affected by Pin 1 depletion (Figure 3, K and L). Together, the Pin 1 knockdown and overexpression experiments indicate that Pin 1 specifically decreases steady-state PTH mRNA levels and PTH mRNA stability by IVDA and this is dependent upon the PTH mRNA ARE.

To determine whether the regulation of PTH mRNA levels by Pin 1 is mediated by the PTH mRNA ARE in the transfected cells, we used a GH reporter gene containing the rat PTH 63-nt ARE within its 3' UTR (GH63) (Figure 4A) (11). As in our previous reports, the PTH mRNA ARE decreased GH63 mRNA levels compared with WT GH mRNA (Figure 4B) (4). Pin 1 overexpression led to a further decrease in chimeric GH63 mRNA levels but had no effect on WT GH mRNA levels (Figure 4, B and C). We then studied the effect of the Pin 1 inhibitor juglone on GH63 or WT GH. Juglone decreased endogenous Pin 1 protein levels in HEK293 cells, as expected (Figure 4D). Importantly, juglone increased GH63 mRNA levels compared with control cells treated with vehicle but had no effect on WT GH mRNA levels (Figure 4, E and F). Together, our results indicate that Pin 1 decreases steady-state PTH mRNA levels, through the PTH mRNA ARE. 
A

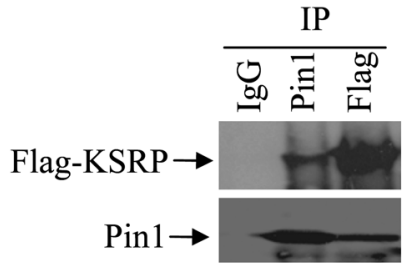

B

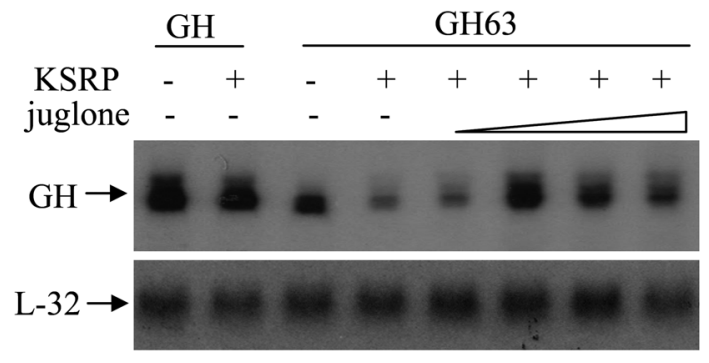

D
C

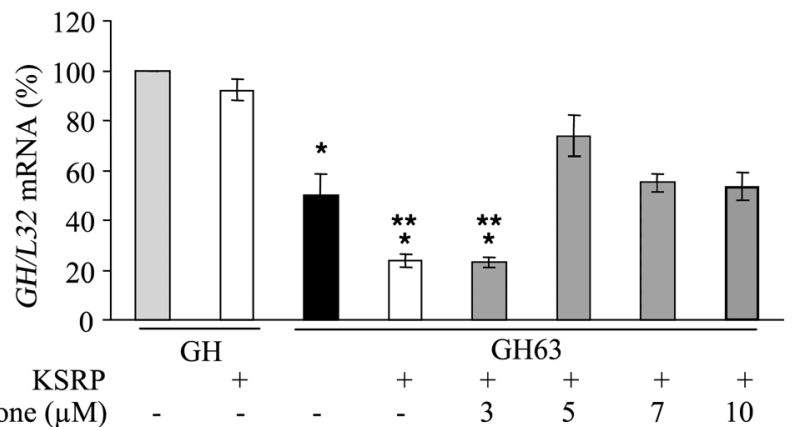

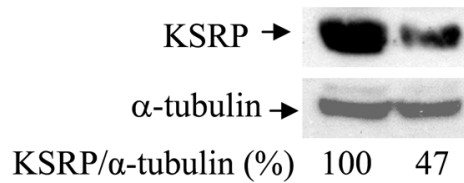

E

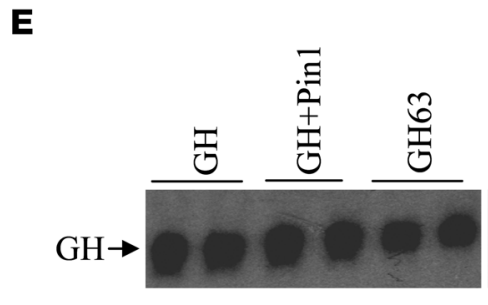

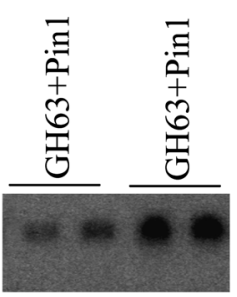

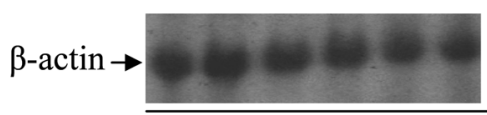

siRNA

CAT

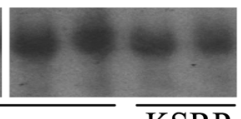

$\overline{\text { KSRP }}$

$\mathbf{F}$

\section{Figure 5}

Pin1 interacts with the PTH mRNA decay-promoting protein KSRP, and the effects of Pin1 and KSRP on the PTH mRNA ARE are interdependent. (A) Pin1-KSRP interaction. HEK293 cells were transiently transfected with FLAG-KSRP plasmid and extracts immunoprecipitated with anti-FLAG or anti-Pin1 antibodies or IgG, run on SDS PAGE, and immunoblotted with the corresponding antibodies. (B and C) Cells were cotransfected with either GH or GH63 and KSRP or control pcDNA3 plasmids. After 48 hours, increasing concentrations of juglone were added for an additional 4 hours. (B) Northern blots for GH mRNA. (C) Quantification of 3 Northern blots as in B; ${ }^{*} P<0.05$ compared with GH mRNA without KSRP; ${ }^{* *} P<0.05$ compared with GH63 mRNA without KSRP. (D-F) KSRP knockdown. Cells were cotransfected with either GH or GH63 and GFP-Pin1 or control pcDNA3 plasmids together with either CAT or KSRP siRNAs. (D) Immunoblots for KSRP and $\alpha$-tubulin protein levels 48 hours after transfection, showing decreased KSRP in KSRP siRNA-transfected cells. Densitometry analysis of KSRP corrected for $\alpha$-tubulin is shown below the gels. (E) Northern blots for GH mRNAs. The lanes were run on the same gel but were noncontiguous (white line). (F) Quantification of 3 Northern blots as in $\mathbf{E}$ for GH (left) and GH63 (right); ${ }^{*} P<0.05$. Data in $\mathbf{C}$ and $\mathbf{F}$ are represented as mean \pm SEM.

Pin1 interacts with the PTH mRNA decay-promoting protein KSRP, and the effects of KSRP and Pin1 on PTH mRNA are interdependent. $P T H$ mRNA stability is regulated by the coordinated interaction of AUF1 and KSRP with the same PTH mRNA 3' UTR 63-nt ARE (11). AUF1 interacts with Pin1, resulting in AUF1 isomerization and hyperphosphorylation upon Pin 1 activation (20). KSRP-Pin1 interaction has not been reported. To study such interaction, we performed coimmunoprecipitation analysis of FLAG-KSRP-transfected HEK293 cell extracts. Endogenous Pin 1 was specifically immunoprecipitated by FLAG-KSRP (Figure 5A). Similarly, Pin1 immunoprecipitated FLAG-KSRP in these extracts (Figure 5A).

To determine the functional importance of KSRP-Pin 1 interaction, we studied the effect of Pin 1 inhibition by juglone on the
KSRP-mediated regulation of PTH gene expression in transfected cells. For that, HEK293 cells were transiently cotransfected with expression plasmids for the GH63 reporter gene or WT GH and either a control or KSRP expression plasmid. KSRP decreased GH63-nt mRNA levels while having no effect on WT GH mRNA (Figure 5, B and C), as in our previous studies (11). Pin 1 inhibition by juglone specifically prevented the KSRP-mediated decrease in GH63 mRNA levels and restored GH63-nt mRNA levels to those of control cells. There was no effect of either KSRP or juglone on WT GH mRNA levels (Figure 5, B and C).

We then studied the effect of KSRP depletion by siRNA on the regulation of GH63 mRNA levels by Pin 1 overexpression. HEK293 cells were cotransfected with GH or GH63-nt and either a control 
A

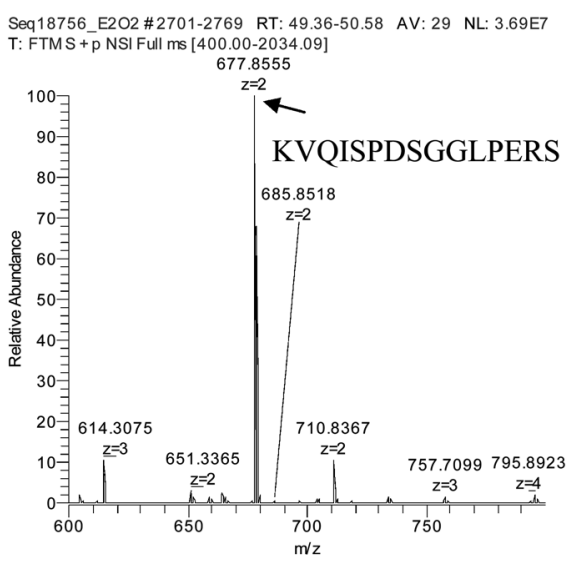

Seq18756 E2O2\#2701-2769 RT: 49.36-50.58 AV: 29 NL: $3.69 E 7$

Seq18756_E2O2\#2819-2855 RT: 51.54-52.15 AV: 15 NL: 5.77E5 T: FTM S + p NSI Full ms [400.00-2034.09]
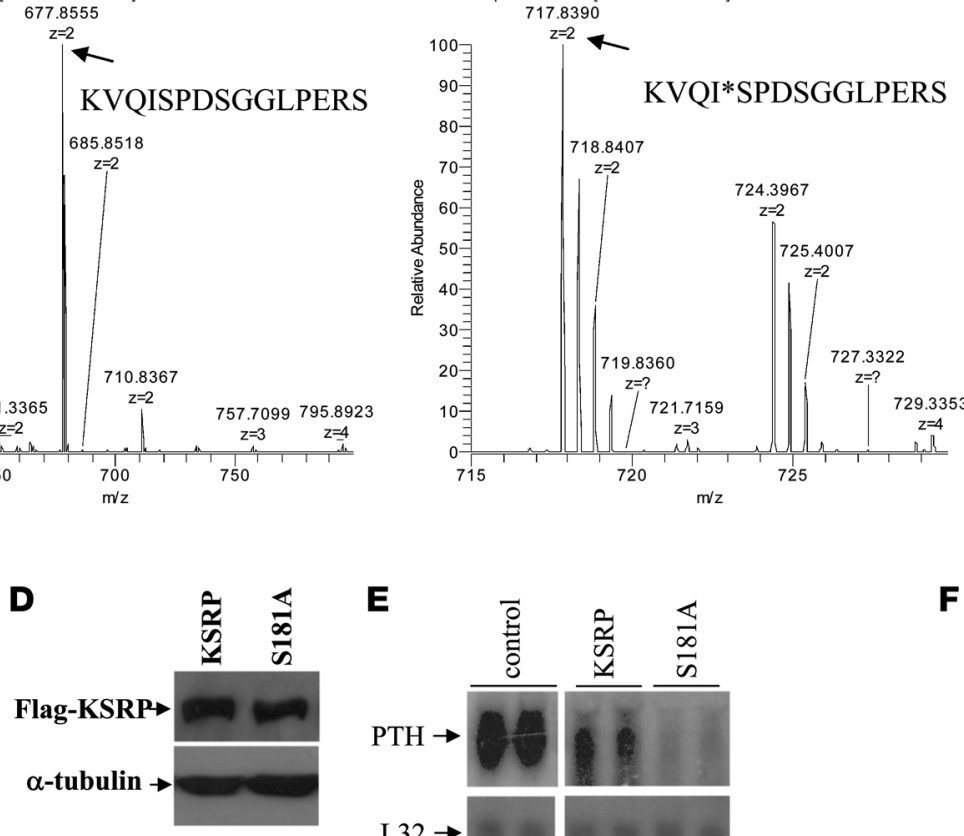

$\mathbf{E}$
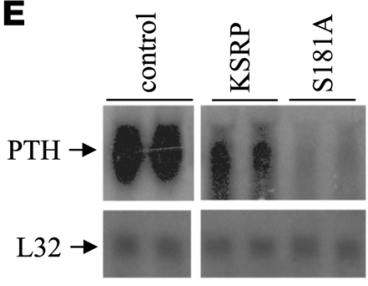

G
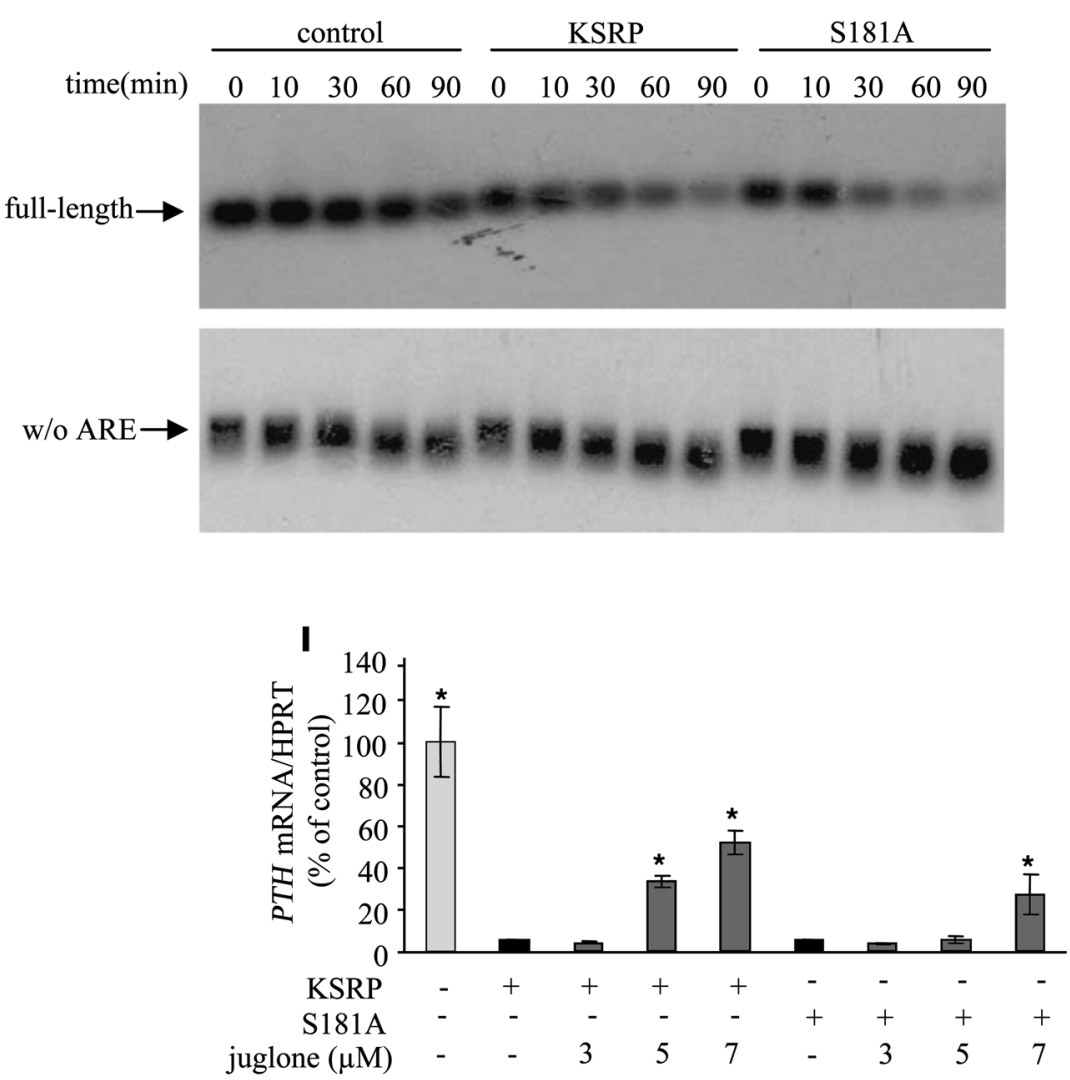

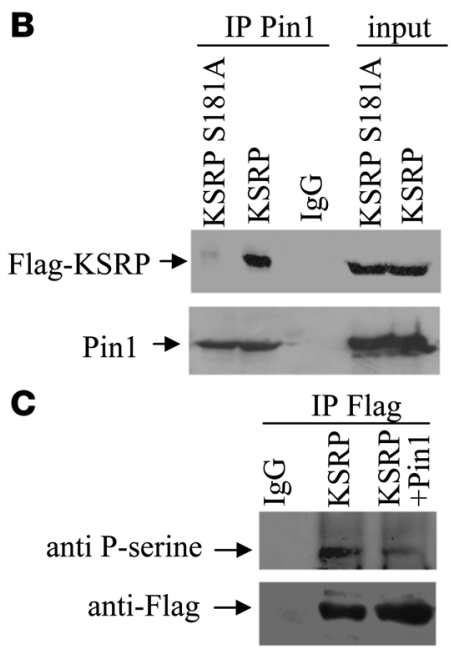

F

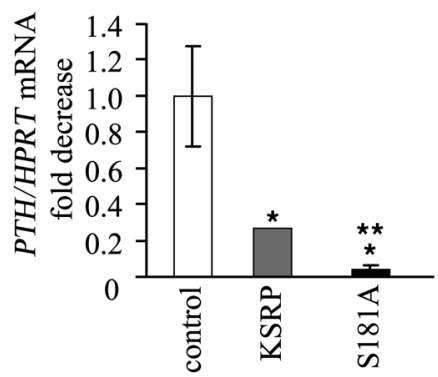




\section{Figure 6}

Identification of KSRP phosphorylation at S181 and its role in KSRPPin1 interaction and PTH mRNA regulation. (A) Mass spectrometry. MS spectra of tryptic mixture of immunoprecipitated FLAG-KSRP from transfected cells. The peak at $677.8555 \mathrm{~m} / \mathrm{z}$ was assigned as the unphosphorylated form of amino acids 177-197 (left), and the peak at $717.8390 \mathrm{~m} / \mathrm{z}$ the phosphorylated form of the peptide (right). Asterisk indicates serine phosphorylation. The $\mathrm{P}$ probability of the assignment was equal to 7.51E-06. Sequest $X$ score was equal to 3.25. (B) KSRPPin1 interaction. HEK293 cells were cotransfected with either FLAGKSRP or FLAG-KSRP S181A and GFP-Pin1 or control plasmid. Extracts were immunoprecipitated with either anti-Pin1 or lgG. The pellets and input were immunoblotted as indicated. (C) KSRP dephosphorylation. Cells were cotransfected with FLAG-KSRP and GFP-Pin1 or control plasmid. Extracts were immunoprecipitated with anti-FLAG or IgG and immunoblotted with anti-phosphor-serine or anti-FLAG antibody. (D-H) Cells were cotransfected with the PTH plasmid or control and either FLAG-KSRP or FLAG-KSRP S181A. (D) Immunoblots showing equal amounts of KSRP. (E) Northern blots of PTH mRNA. The lanes were run on the same gel but were noncontiguous (white line). (F) qRT-PCR for PTH mRNA as in E. *Compared with control; ${ }^{*}$ compared with KSRP. (G) IVDA using cell extracts and full-length human PTH mRNA (top) or PTH mRNA without the ARE (bottom). (H) Quantification of IVDA results in 3 experiments; mean \pm SEM of time 0; KSRP compared with both control and S181A; ${ }^{*} P<0.05$. (I) Cells were cotransfected with the PTH and KSRP or S181A plasmids with and without juglone. RNA was analyzed by qRT-PCR for PTH mRNA; ${ }^{*} P<0.05(n=3)$ compared with cells expressing KSRP and not treated with juglone.

or a Pin1 expression plasmid (Figure 5, D-F). Pin1 overexpression decreased GH63-nt mRNA levels while having no effect on the control GH mRNA as before (Figure 5, E and F, and Figure 4, B and C). siRNA-mediated knockdown of KSRP by approximately $50 \%$ (Figure 5D) caused a specific 3-fold increase in GH63-nt mRNA levels compared with control siRNAs (not shown) as before (11). Importantly, the effect of Pin 1 in decreasing GH63 mRNA levels was attenuated in the KSRP-depleted HEK293 cells (Figure 5, E and F). Together, these results indicate that Pin 1 and KSRP act together to regulate PTH mRNA levels.

KSRP is phosphorylated at S181, and Pin1 overexpression induces dephosphorylation of KSRP at serine residues. KSRP is a phosphoprotein with 2 reported phosphorylation sites, at S193 and T692 (26). To identify a Pin 1 interaction consensus site on KSRP, we transfected HEK293 cells with FLAG-KSRP and analyzed immunoprecipitated KSRP by mass spectrometry. This analysis identified a new phosphorylation site at S181 in the KVQI*SPDSGGLPER peptide that has not been previously reported (Figure 6A and Supplemental Figure 1; supplemental material available online with this article; doi:10.1172/ JCI39522DS1). This serine residue is adjacent to proline, making it a putative Pin1-interacting site. To study the role of S181 on KSRP-Pin1 interaction, we cotransfected HEK293 cells with expression plasmids for Pin 1 and either FLAG-KSRP or the FLAG-KSRP S181A mutant. Cotransfected Pin1 did not immunoprecipitate FLAG-KSRP S181A but did precipitate FLAG-KSRP (Figure 6B and Figure 5A). In contrast, both KSRP S193A and T692A mutants bound Pin 1 as the WT KSRP (Supplemental Figure 2, A and B).

We then studied the effect of Pin 1 overexpression on KSRP phosphorylation. HEK293 cells were transfected with FLAGKSRP and a Pin 1 expression plasmid. Immunoprecipitated FLAG-KSRP was analyzed by immunoblots with anti-phosphoserine antibody. Pin 1 overexpression decreased the amount of phosphorylated KSRP at serine residues (Figure 6C). Therefore,
Pin 1 decreases KSRP phosphorylation at serine residues, and an intact serine at amino acid 181 but not at 193 is necessary for Pin1-KSRP interaction in transfected cells.

S181A KSRP mutant is more potent than WT KSRP in decreasing PTH $m R N A$ levels in transfected cells. KSRP phosphorylation at S193 by AKT prevents the binding of KSRP to the exosome complex and thereby KSRP-induced mRNA decay (27). We studied the effect of the newly identified S181 phosphorylation site on KSRP-mediated PTH mRNA degradation. HEK293 cells were cotransfected with expression plasmids for PTH and either WT or S181A KSRP. Both WT and S181A KSRP were expressed at the same levels (Figure 6D). WT KSRP overexpression decreased PTH mRNA levels (Figure 6, E and F) as before (26). The overexpressed S181A mutant was more potent than WT KSRP (Figure 6, E and F). These results were confirmed by IVDAs using extracts from HEK293 cells transfected with either KSRP or KSRP S181A mutant and uniformly labeled PTH mRNA as above. As expected, overexpression of KSRP specifically decreased $P T H$ mRNA stability compared with control extracts (Figure 6, G and H). PTH mRNA decay was further accelerated by cell extracts overexpressing KSRP S181A compared with extracts expressing WT KSRP (Figure 6, G and H). There was no difference in mRNA decay of the truncated PTH transcript lacking the PTH mRNA ARE (Figure 6, G and H). These results demonstrate that dephosphorylated KSRP at the Pin1 interaction site, S181, is more potent than WT KSRP in decreasing PTH mRNA levels and stability. They suggest that Pin 1 acts through dephosphorylation of KSRP to decrease PTH mRNA levels in an AREdependent manner in transfected cells.

We therefore hypothesized that S181A KSRP, which does not bind Pin1, would not be influenced by Pin 1 inhibition. HEK293 cells were transiently cotransfected with expression plasmids for PTH and either WT or S181A KSRP and were incubated with juglone, as above (Figure 4, E and F). KSRP overexpression caused a 90\% reduction in PTH mRNA levels (Figure 6I). Juglone treatment prevented this decrease dose dependently (Figure 6I), similar to its effect on reporter GH63 (Figure 4, E and F). In contrast, juglone had no effect on PTH mRNA levels at 3 and $5 \mu \mathrm{M}$ in cells expressing S181A KSRP and only an effect at the highest juglone concentration (Figure 6I). Together, these results indicate that Pin1-KSRP interaction is dependent upon S181 KSRP phosphorylation, suggesting that Pin1 leads to dephosphorylation at this site and thereby activation of KSRP.

Pin1 interacts with KSRP and regulates KSRP-PTH $m R N A$ interaction in parathyroid glands. In vivo in the parathyroid, Pin 1 activity was decreased in parathyroid extracts of rats with increased PTH mRNA levels and stability (Figure 1), and Pin 1 inhibition by juglone increased $P T H$ mRNA levels posttranscriptionally (Figure 2 ). We have previously shown that posttranscriptional regulation of PTH gene expression is mediated by differential binding of KSRP to the PTH mRNA ARE (11). To study KSRP-PTH mRNAPin 1 interactions in vivo, we first showed KSRP-Pin 1 interaction in rat parathyroid gland extracts (Figure 7A). KSRP was coimmunoprecipitated by Pin 1 (Figure 7A), indicating that these proteins interact also in the parathyroid, similar to their interaction in HEK293 cells (Figure 5A).

We then asked whether the effect of juglone to increase PTH mRNA levels is mediated by an effect of Pin 1 on KSRP-PTH mRNA interaction in vivo. Juglone was applied topically to rat parathyroid glands as above (Figure 2, $\mathrm{C}-\mathrm{F}$ ). The parathyroid glands were then cross-linked and analyzed by RNA immunoprecipitation 
A

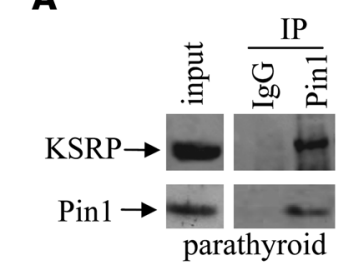

C

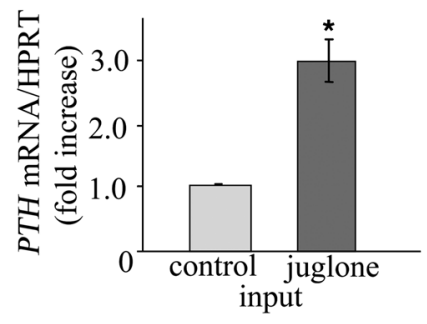

B

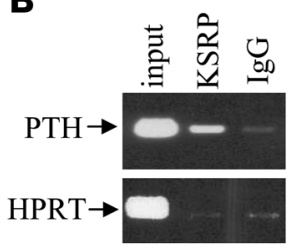

D

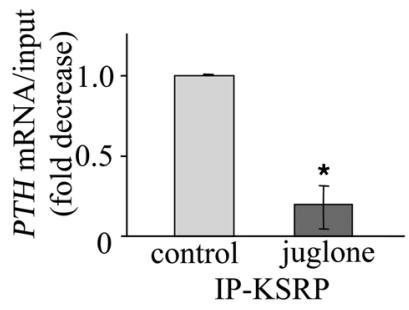

Figure 7

Pin1 interacts with KSRP and regulates KSRP-PTH mRNA interaction in parathyroid glands. (A) Pin1-KSRP interaction in the parathyroid. Pin1 was immunoprecipitated from microdissected parathyroid extracts using anti-Pin1 antibody or IgG, fractionated on SDS PAGE, and analyzed by immunoblots for KSRP and Pin1. The lanes were run on the same gel but were noncontiguous (white line). (B-D) RIP analysis. Parathyroid glands were subjected to juglone or vehicle applied topically. RIP was performed on rat parathyroid glands using either anti-KSRP or control IgG. RNA from total lysate (input) and KSRP (IP-KSRP) or control IgG-bound fractions was assayed by qRT-PCR for PTH and control HPRT mRNAs. (B) Agarose gel of qRT-PCR products for PTH and HPRT. The size of PCR products was consistent with the predicted lengths of the amplified fragments. (C) PTH mRNA levels in the input. (D) mRNA levels in immunoprecipitated samples, corrected for mRNA levels in input; mean \pm SEM of 3 experiments using parathyroids from pools of different rats. (E) Immunoblots of the parathyroid extracts as in $\mathbf{C}$ (input). ${ }^{*} P<0.05$.

(RIP) using anti-KSRP antibody and qRT-PCR analysis. As shown in Figure 7B, RIP analysis showed a specific interaction of KSRP with PTH mRNA and not with a control HPRT mRNA, as we have previously published (11). Juglone increased PTH mRNA levels in the input fraction (Figure 7C) as above (Figure 2, E and F). Pin 1 inhibition led to a marked decrease in the amount of KSRPbound PTH mRNA compared with vehicle-treated parathyroid glands (Figure 7D). This decrease in KSRP-PTH mRNA interaction was not a result of changes in KSRP protein levels that were unchanged by juglone treatment (Figure 7E). Juglone led to the expected decrease in Pin 1 protein levels (Figure 7E) (23). AUF1 protein levels were also dramatically decreased in the juglone-treated parathyroid glands, as reported by Shen et al. $(20,21)$. Due to the marked decrease in AUF1 protein levels here, it was not possible to study AUF1-PTH mRNA interactions in the juglone-treated parathyroids. Our results indicate that Pin 1 inhibition by juglone in vivo decreases KSRP-PTH mRNA interaction in the parathyroid. This may contribute to the increased $P T H$ mRNA levels induced by juglone (Figure 7C and Figure 2, E and F).

Pin $^{-1-}$ mice have increased PTH expression. Our results indicate a correlation between Pin 1 activity and $P T H$ mRNA levels in vivo and regulation of $P T H$ gene expression by Pin 1 in vitro. To study the role of Pin 1 in PTH gene expression in vivo more directly, we measured serum PTH, calcium, and phosphorus levels in mice with genetic deletion of Pin1 (Pin ${ }^{-/}$mice) (28). Serum PTH levels in the $\mathrm{Pin}^{-/-}$mice were 3 -fold higher than in WT littermates (Figure $8 \mathrm{~A})$. There was no change in serum calcium and phosphorus lev-
$\mathbf{E}$

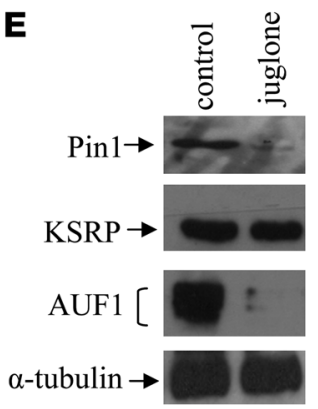

els (Figure 8, B and C) nor in CYP27B1 (1 $\alpha$-hydroxylase) mRNA levels (Supplemental Figure 3). Therefore, the increased serum PTH was not secondary to changes in serum calcium or phosphorus levels. Immunohistochemistry using an anti-PTH antibody demonstrated a 2-fold increase in PTH protein content in the parathyroid gland of the $\mathrm{Pin}^{-/-}$ mice compared with control WT littermates (Figure 8, D and E), in agreement with the high-serum PTH levels in the Pin $1^{-/-}$mice. Similarly, in situ hybridization demonstrated an approximately 2 -fold increase in PTH mRNA levels in the parathyroid gland of the $\mathrm{Pin}^{-/-}$mice (Figure 8, F and G). Together, these results indicate that Pin 1 determines basal PTH levels in vivo.

\section{Discussion}

The increased PTH mRNA levels of secondary hyperparathyroidism due to calcium depletion or CKD is mediated by the interaction of trans-acting RNAbinding proteins with a defined cis-acting ARE in the PTH mRNA 3' UTR. AUF1 and Unr (upstream of N-ras) stabilize and KSRP destabilizes the PTH mRNA (11, 22). Many RNA-binding proteins, including KSRP and AUF1, are phosphoproteins, and their activity is tightly regulated through phosphorylation and dephosphorylation signaling cascades. In particular, AUF1 is posttranslationally modified in the parathyroid in correlation with $P T H$ gene expression in secondary hyperparathyroidism (29). Here, we demonstrate for what we believe is the first time that Pin 1 is a critical mediator of PTH expression in vivo and in vitro in transfected cells. Pin $1^{-/-}$mice have increased levels of serum PTH, parathyroid PTH protein, and PTH mRNA levels, indicating that Pin 1 determines basal PTH expression in vivo. Moreover, Pin 1 isomerase activity is decreased in parathyroid extracts of rats fed a calcium-depleted diet or CKD rats in which PTH mRNA levels and stability are increased. Juglone inhibits Pin 1 activity and leads to its degradation by the proteasome (20). Juglone has also recently been shown to inhibit postmitotic dephosphorylation and the exit of mitosis independently of Pin 1 by covalent modification of sulfhydryl groups in proteins essential for metaphase/anaphase transition (30). We show that Pin inhibition by juglone increases PTH mRNA levels in vivo posttranscriptionally. The effect of juglone occurs when it is applied topically on the parathyroid or given systemically, indicating a direct effect of the Pin 1 inhibitor on the parathyroid. We used HEK293 cells, which reproduce certain aspects of the regulation of a transfected $P T H$ gene, to study the effect of Pin 1 on PTH gene expression $(11,31,32)$. Pin 1 regulates $P T H$ gene expression in transiently transfected HEK293 cells, and this regulation is dependent upon the PTH mRNA 3' UTR ARE and KSRP. We identify KSRP as a new target protein for Pin1. Pin 1 interacts with KSRP and leads to KSRP dephosphorylation, which enhances the KSRP-mediated decrease in PTH mRNA levels. 
A

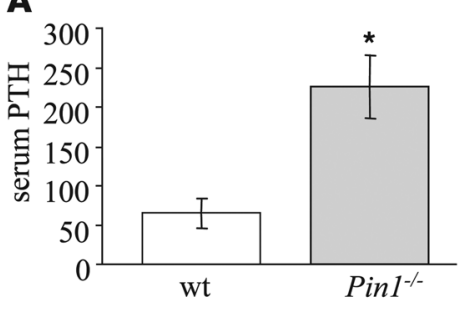

D

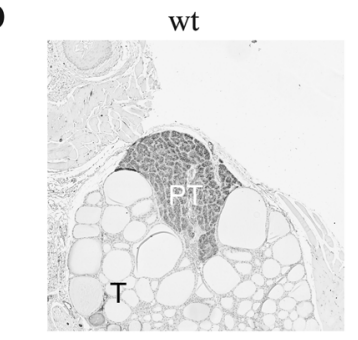

F

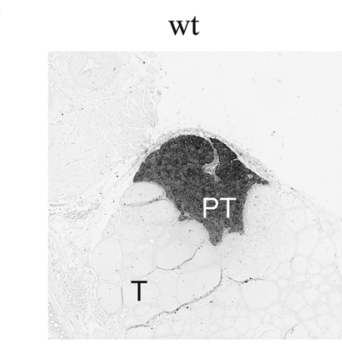

B

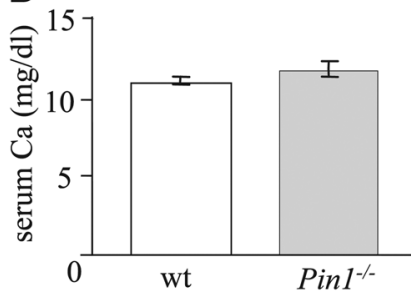

C

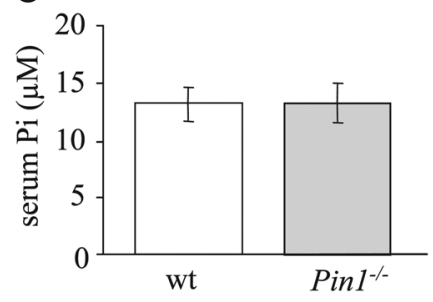

E
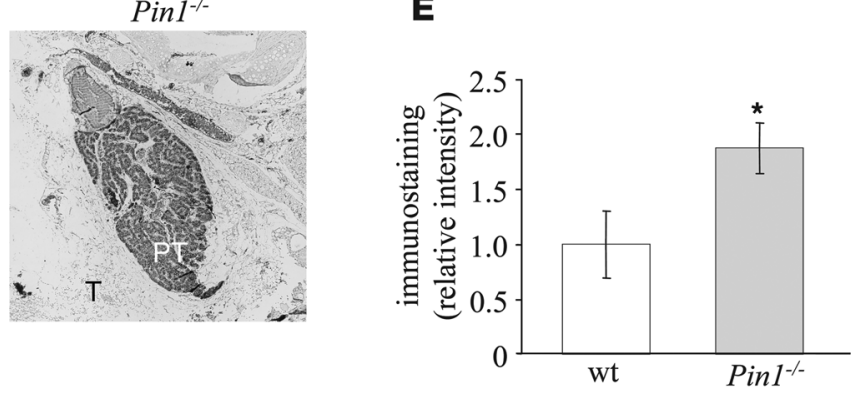

G

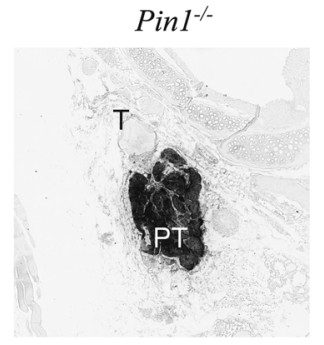

Figure 8

$\mathrm{Pin}^{-/-}$mice have high serum PTH and PTH mRNA levels. (A-C) Serum PTH, calcium, and phosphate levels in WT $(n=18)$ and Pin1 $1^{-/-}(n=14)$ mice. (D) Immunostaining of thyroparathyroid section for PTH. (E) Quantification of data presented in 6 sections from each group. (F) In situ hybridization for PTH mRNA in thyroparathyroid sections. (G) Quantification of the in situ hybridization staining in 6 sections from each group. Original magnification, $\times 100(\mathbf{D}$ and $\mathbf{F}) .{ }^{*} P<0.05$. PT, parathyroid; $\mathrm{T}$, thyroid tissue.

In vivo, Pin 1 inhibition decreases KSRP-PTH mRNA interaction, which would lead to the increased PTH mRNA and serum PTH levels after juglone administration.

The Pin $1^{-1-}$ mice have high serum PTH and PTH mRNA levels without the expected hypercalcemia or hypophosphatemia or increase in CYP27B1 ( $1 \alpha$-hydroxylase) mRNA levels. This may represent downregulation of the $\mathrm{PTH}$ receptor in these knockout mice. In fact, the bones of Pin $1^{-/-}$mice are markedly abnormal, which was attributed to a premature aging phenotype and not hyperparathyroidism (33).

It was recently shown in HeLa cells that Pin 1 shuttles between nuclear and cytoplasmic domains. A nuclear localization signal located at the PPIase domain is based on 3 residues: Lys63, Arg68, and Arg69 (34). Interestingly, our results indicate that even though mutation at Lys63 did not affect Pin 1 activity, double-point mutation at Arg68 and Arg69 residues dramatically disrupted Pin 1 activity on PTH mRNA levels. The effect of the R68/69 mutation on Pin 1 activity here may be due to damage to the Pin 1 PPIase domain itself by the mutation. The WW-binding domain mutation (W34A) functions as a dominant negative mutation by preventing endogenous Pin1 from binding to Ser/Thr-Pro targets (35). Mutation at the WW domain attenuated the decrease in PTH mRNA levels by Pin1. Together, our results suggest that both the Pin1 PPIase and WW domains are necessary for its effect on PTH mRNA levels.
AUF1 was shown to be a Pin 1 target protein $(20,21)$. AUF1 consists of 4 isoforms: p37, p40, p42, and p45 (36). Pin 1 inhibition by juglone in eosinophil cells leads to proteosome-mediated degradation of 3 of the 4 AUF1 isoforms, p40, p42, and p45, while isoform p37 remains stable and destabilizes TGF- $\beta$ and GM-CSF mRNA (20, $21)$. The stabilizing action of AUF1 on the PTH mRNA is in contrast to its effect on many other ARE-containing mRNAs in which it is a destabilizing factor $(10,37)$. In the parathyroid, juglone led to degradation of all 4 AUF1 isoforms to undetectable levels (Figure $7 \mathrm{E}$ ). We could therefore not determine a contribution of any of the AUF1 isoforms to the effect of Pin 1 on PTH gene expression. It is paradoxical that juglone increased $P T H$ mRNA levels both in transfected cells and in the parathyroid while decreasing protein levels of AUF1, which is a PTH mRNA-stabilizing protein. It is possible that small amounts of AUF1 that remain bind with a higher affinity to the PTH mRNA 3' UTR and stabilize the PTH mRNA.

We show that the effect of KSRP in decreasing PTH gene expression is dependent upon Pin1. Moreover, Pin 1 inhibition by juglone prevents KSRP-mediated $P T H$ mRNA decay through the $P T H$ mRNA ARE and decreases KSRP-PTH mRNA interaction in vivo with no change in KSRP protein levels. KSRP is a phosphoprotein with 2 characterized phosphorylation sites, S193 and T692 (26, 27). T692 is adjacent to a proline, making it a putative consensus Pin1interacting site. Mutations at these 2 sites did not interfere with 


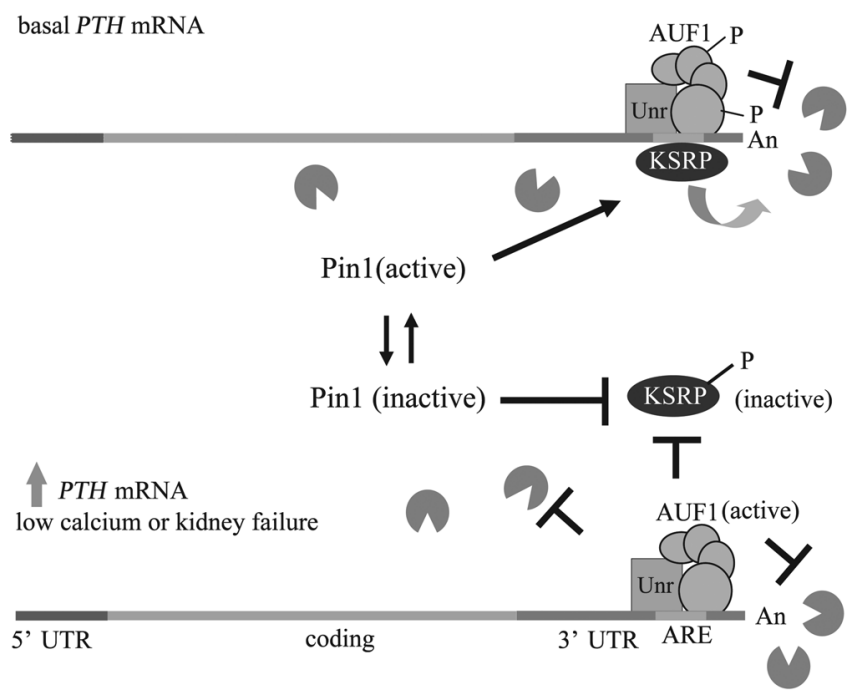

Figure 9

Model for the regulation of PTH mRNA stability by PTH mRNA 3 UTR ARE binding proteins. Under basal conditions, there is a balanced interaction of the PTH mRNA with its stabilizing proteins AUF1 (isoforms p37, p40, p42 and p45) and Unr and the destabilizing protein KSRP. In hypocalcemia or CKD, Pin1 is inactive, resulting in KSRP phosphorylation and hence its inactivation. This would allow AUF1 and Unr to bind the PTH mRNA 3' UTR ARE with a greater affinity, leading to increased $P T H$ mRNA stability.

KSRP-Pin 1 interaction, indicating that these 2 phosphorylated sites are not the Pin1-interacting sites. We identify a new phosphorylation site at S181 in the N terminus of the protein. S181 is adjacent to proline, forming a potential Pin 1 interaction site. Indeed, the S181A mutation prevented Pin1-KSRP interaction and enhanced PTH mRNA destabilization by KSRP compared with WT KSRP. Moreover, Pin1 inhibition by juglone did not affect the decrease in PTH mRNA levels by KSRP S181A.

Our results suggest that phosphorylated KSRP at S181 is inactive. Upon interaction with Pin1, cis-trans isomerization of the proline bond in KSRP leads to conformational change, exposing the phosphorylated S181 residue and possibly additional phosphorylation sites. This leads to dephosphorylation, thus activating KSRP, which then interacts with PTH mRNA and enhances its decay (Figure 9). A low calcium diet and CKD led to decreased Pin1 isomerase activity in the parathyroids of these rats. This decreased Pin 1 activity would prevent KSRP dephosphorylation, resulting in decreased KSRP-PTH mRNA interaction, inhibition of PTH mRNA degradation, and increased PTH mRNA levels. Consistent with this finding, Pin 1 inhibition by juglone increases $P T H$ mRNA levels and decreases KSRP-PTH mRNA interaction. Pin1 is therefore a regulator of $P T H$ gene expression. Pin 1 determines basal PTH levels and the response of the parathyroid to chronic hypocalcemia and CKD.

\section{Methods}

Animals. Weanling male Sabra rats were fed either control or calciumdepleted diets (Teklad) for 2 weeks (2). Calcium depletion results in hypocalcemia (controls, $10.5 \pm 0.4$; low calcium, $4.8 \pm 0.5 \mathrm{mg} / \mathrm{dl} ; n=10)$ and increased serum PTH (controls, $56 \pm 12$; low calcium, $632 \pm 57 \mathrm{pg} / \mathrm{ml}$ ). Adult male rats $(100-120 \mathrm{~g})$ were fed a control diet or a $0.75 \%$ adenine, high-phosphorus (3.0\%) diet for 7 days to induce renal failure (CKD). At 7 days, there was an increase in serum creatinine (controls, $0.27 \pm 0.03 \mu \mathrm{M}$; adenine, $0.7 \pm 0.08 \mu \mathrm{M} ; n=10$ ), no change in serum phosphate (controls, $10.9 \pm 0.25$; adenine, $9.9 \pm 0.3 \mathrm{mg} / \mathrm{dl}$ ), and increase in serum PTH (controls, $66 \pm 22 \mathrm{pg} / \mathrm{ml}$; adenine, $338 \pm 113$ ). Serum biochemistry for calcium, phosphorus, and creatinine were measured using a QuantiChrom Calcium Phosphate Assay Kit (BioAssay Systems). Serum rat intact PTH levels were measured using the intact PTH ELISA Kits (Immunotopics).

For systemicjuglone admission, rats were injected i.p. with juglone $(1 \mathrm{~g} / 1 \mathrm{~kg} / \mathrm{d})$ or a vehicle (ethanol) for 2 days, and rats were sacrificed 1 hour after the second injection. For topical juglone administration, the parathyroid glands of anesthetized rats were exposed and submerged in DMEM containing either $5 \mu \mathrm{M}$ juglone or vehicle (ethanol, diluted 1:104) for 2 hours. All animal experiments were approved by the Institutional Animal Care and Use Committees of the Hadassah Hebrew University and Tohoku University.

Pin 1 activity assay. Pin 1 assay was performed as described (23). Microdissected parathyroid glands (pool of 5) were placed on ice in a reaction buffer containing $100 \mathrm{mM} \mathrm{NaCl}, 50 \mathrm{mM}$ HEPES, pH 7, 2 mM DTT, and $0.04 \mathrm{mg} / \mathrm{ml}$ BSA. The glands were homogenized using a mini-bead beater (Biospec Products) and the supernatant cleared by centrifugation at $12,000 \mathrm{~g}$ for 10 minutes $\left(4^{\circ} \mathrm{C}\right)$. PPIase activity was measured using equal amounts of parathyroid cytoplasmic lysates and $\alpha$-chymotrypsin using a synthetic tetrapeptide substrate Suc-Ala-Glu-Pro-Phe-pNa (Peptides International). Absorption at $390 \mathrm{nM}$ was measured using an Ultrospec 2000 spectrophotometer. The results are expressed as the mean of 3 measurements from a single experiment and are representative of 3 independent experiments.

RNase protection assay. RNA was extracted from microdissected parathyroid glands (pool of 5), and equal amounts of RNA were analyzed by ${ }^{32}$ P UTPlabeled reverse RNA probes. The intact probes before and after RNase treatment and hybridization with tRNA used as a nonspecific control were included in each experiment. The templates for the antisense RNA probes were generated by PCR using a forward primer with an upstream T3 promoter sequence. The primers for the PTH pre-mRNA first intron sequence were as follows: $5^{\prime}$-ATTAACCCTCACTAAAGGGTTTCCAAGCATTCACCATCA- $3^{\prime}$ and $5^{\prime}$-CCTTTGAAACTCTCCCAACG- $3^{\prime}$. For the GAPDH mRNA, sequences were as follows: 3' UTR: 5'-GTGGACCTCATGGCCTACAT-3' and $5^{\prime}$-ATTAACCCTCACTAAAGTGTGAGGGAGATGCT-3'. The antisense probe for the $P T H$ mRNA $3^{\prime}$ UTR was transcribed from a linearized plasmid containing the rat PTH $3^{\prime}$ UTR using T7 RNA polymerase (10).

$R N A$ transcription and labeling. Uniformly $\left[\alpha^{-32} \mathrm{P}\right] \mathrm{UTP}-\mathrm{labeled}$ transcripts were prepared as previously described (11). A transcript for the full-length mRNA was transcribed from either the full-length human PTH cDNA or the full-length rat PTH cDNA (11). Transcripts for the truncated human PTH mRNA without the $3^{\prime}$ UTR or the rat PTH mRNA with an internal deletion of the ARE ( $90 \mathrm{nt}$ ) were also used (11). The resulting templates of the rat PTH cDNAs contained a stretch of approximately $150 \mathrm{bp}$ of $\mathrm{dT}$ at the $3^{\prime}$ end.

IVDAs. Radiolabeled polyadenylated transcripts $(200,000$ counts $/ \mathrm{min})$ were incubated with $40 \mu \mathrm{g}$ protein extracts. At timed intervals, samples were removed; RNA was extracted, separated on agarose gels, and analyzed by autoradiography as previously described (11).

Cell cultures and transient transfection. HEK293 cells were transiently transfected in 24-well plates for RNA analysis and in 10-cm plates for protein extractions, with the indicated plasmids using a Ca phosphate transfection kit (Sigma-Aldrich). siRNA oligonucleotides were transiently cotransfected with expression plasmids, using Lipofectamine 2000 Reagent (Invitrogen). Total amount of DNA in cotransfection experiments was maintained constant using an empty vector, as indicated. In some experiments, juglone (3-7 $\mu \mathrm{M}$ ) or vehicle (ethanol) were added 48 hours after transfection for an additional 4 hours. 
Northern blots and immunoblots. RNA was extracted using peqGOLD TriFast (PeqLab Biotechnologie) and analyzed as previously described (11). For immunoblots, proteins were analyzed by SDS-PAGE as previously described (11).

Protein extractions. For IVDAs, postmitochondrial extracts were prepared from cultured cells as before (11). Parathyroid extracts were prepared similarly from a pool of 5 rats in each group. For Western blots and RIP, cultured cells or parathyroid glands (pool of 5 rats) were prepared using RIPA buffer containing $150 \mathrm{mM} \mathrm{NaCl}, 1 \% \mathrm{NP} 40,0.5 \%$ sodium deoxycholate, $0.1 \% \mathrm{SDS}$, and protease inhibitors (11). Extracts were stored in aliquots at $-80^{\circ} \mathrm{C}$.

Antibodies. Anti-KSRP was a generous gift from R. Gherzi (Istituto Nazionale per la Ricerca sul Cancro, Genova, Italy). The anti-FLAG, anti- $\alpha$-tubulin, and anti-phosphoserine were from Sigma-Aldrich. Anti-GAPDH and anti-Pin1 were from Santa Cruz Biotechnology Inc. Anti-PTH monoclonal antibody used for immunohistochemistry was from AbD Serotec.

Plasmids. The human PTH gene, including exons and introns, was in pcDNA3 (11). FLAG-KSRP in pcDNA3 contained FLAG-tagged full-length KSRP, KSRP S193A, or T692A and was kindly provided by R. Gherzi. The GH expression plasmid was kindly provided by O. Meyuhas (Hebrew University-Hadassah Medical School, Jerusalem, Israel). The GH-PTH mRNA 63-nt plasmid was previously described (5) and contained the 63-nt rat PTH mRNA ARE cloned between the 3' of the GH mRNA coding sequence and the GH mRNA 3' UTR. Empty control vector pcDNA3 (Invitrogen) was used as indicated. GFP-Pin1, Pin1 K63A, and Pin1 W34A expression plasmids were kindly provided by K.P. Lu (Department of Medicine, Beth Israel Deaconess Medical Center, Harvard Medical School, Boston, MA, USA) (38) and Pin1 R68/69A by S. Ferrari (Institute of Molecular Cancer Research, University of Zürich, Zürich, Switzerland) (34).

siRNAs. siRNAs targeting the control CAT sequence were as follows: $5^{\prime}$ r(GACGGUGAGCUGGUGAUAU)d(TT)-3', KSRP: 5'-AAGATCAACCGGAGAGCAAGA-3' and Pin1: r(CCGCCAGAUUCUCCCUUAA)d(TT). All were from QIAGEN.

PCR primers for $q R T-P C R$. Rat PTH primers were as follows: 5'TTGTCTCCTTACCCAGGCAGAT- $3^{\prime}$ and $5^{\prime}$-TTTGCCCAGGTTGTGCATAA-3'. Primers for human PTH were as follows: $5^{\prime}$-GGGTCTGCAGTCCAATTCAT- $3^{\prime}$ and $5^{\prime}$-CAGATTTCCCATCCGATTTT- $3^{\prime}$. Primers for rat HPRT were as follows: 5'-TTGGATACAGGCCAGACTTTGTT-3' and 5'-AAGTGCTCATTATAGTCAAGGGCAT-3'. Primers for human HPRT were as follows: 5'-TTTGAATCATGTTTGTGTCATTAGTGA-3' and 5' TTCCAAACTCAACTTGAACTCTCATC-3'.

RIP. RIP was performed as previously described (11). In brief, thyroparathyroid glands (pool of 6) were roughly chopped and cross-linked. Equal amounts of whole-cell extracts were immunoprecipitated with anti-KSRP or IgG as control. RNA was extracted, and equal amounts were reverse transcribed with High Capacity cDNA Reverse Transcription Kit (Applied Biosystems) and analyzed by qPCR and SYBR Green ROX Mix (ABgene). In some experiments, qPCR end products were run on agarose gels.

Site-directed mutagenesis. QuikChange site-directed mutagenesis kit (Stratagene) was used according to the manufacturer's instructions. The primers for KSRP S181A mutagenesis were as follows: 5'-GGCTGCAAAGTACAGATTGCTCCAGACAGCGGTGGCCTACC-3' and 5'-GGTAGGCCACCGCTGTCTGGAGCAATCTGTACTTTGCAGCC-3'.

Nuclear run-on assays. Nuclear run-ons were performed as previously described (2). In brief, nuclei were extracted from parathyroid glands (pool of 5-7). The nuclei were incubated in reaction buffer containing $\left[\alpha_{-}{ }^{32} \mathrm{P}\right]$ UTP for 15 minutes at $37^{\circ} \mathrm{C}$. RNA was extracted using peqGOLD TriFast, and equal amounts of radioactive RNA were hybridized to a nitrocellulose membrane containing linearized and denatured cDNAs for the indicated genes.

Immunobistochemistry and in situ bybridization. Immunohistochemistry was performed as previously described (24). Slides were analyzed using antiPTH antibodies and anti-Pin 1 antibodies or IgG as control. In situ hybridization was performed as previously described (39). For quantification, slides were analyzed by the Ariol SL-50 system (Applied Imaging).

In gel proteolysis and mass spectrometry analysis. The immunoprecipitated proteins were run on an SDS-PAGE, stained, and the proteins from the excised gel slices reduced (10 mM DTT), modified with $40 \mathrm{mM}$ iodoacetamide, and trypsinized (modified trypsin; Promega) at a 1:100 enzyme-to-substrate ratio. The resulting tryptic peptides were resolved by reverse-phase chromatography on $0.075 \times 200-\mathrm{mm}$ fused silica capillaries packed with Reprosil reversed phase material (Dr. Maisch GmbH). The peptides were eluted with linear 65 -minute gradients of $5 \%$ to $45 \%$ and 15 minutes at $95 \%$ acetonitrile with $0.1 \%$ formic acid in water at flow rates of $0.25 \mu \mathrm{l} / \mathrm{min}$. Mass spectrometry was performed by an ion-trap mass spectrometer (Orbitrap; Thermo Scientific) in a positive mode using repetitively full MS scan followed by collision-induced dissociation (CID) of the 7 most dominant ions selected from the first MS scan. The analysis was done with and without multistage activation. The mass spectrometry data were analyzed using Sequest 3.31 software (J. Eng and J. Yates, University of Washington, Seattle, Washington, USA; and Thermo Finnigan) searching against the vertebrate part of the NR-NCBI database.

Statistics. Values are reported as mean \pm SEM unless stated otherwise. A 2-tailed $P$ value was considered significant when less than 0.05 .

\section{Acknowledgments}

We thank R. Gherzi for KSRP plasmids and antibodies and helpful advice; the Smoler Proteomics Center (Haifa Technion, Haifa, Israel) for the MS analysis; Ofra Moshel (Hadassah Hebrew University Medical School, Israel) for help with interpretation of the MS data. This work was supported by an ASBMR bridging fund, the Israel Science Foundation, the Chief Scientist of the Ministry of Health, Israel, and Amgen Pharmaceuticals.

Received for publication April 12, 2009, and accepted in revised form July 15, 2009.

Address correspondence to: Tally Naveh-Many, Minerva Center for Bone and Mineral Metabolism, Nephrology Services, Hadassah University Hospital, PO Box 12000, Jerusalem 91120, Israel. Phone: 972-2-6776789; Fax: 972-2-6421234; E-mail: tally@huji.ac.il.
1. Silver, J., Naveh-Many, T., and Kronenberg, H.M. 2002. Parathyroid hormone: molecular biology. In Principles of bone biology. J.B. Bilezikian, L.G. Raisz, and G.A. Rodan, editors. Academic Press. San Diego, California, USA. 407-422.

2. Moallem, E., Silver, J., Kilav, R., and Naveh-Many, T. 1998. RNA protein binding and post-transcriptional regulation of PTH gene expression by calcium and phosphate. J. Biol. Chem. 273:5253-5259.

3. Levi, R, et al. 2006. Increased parathyroid hormone gene expression in secondary hyperparathyroidism of experimental uremia is reversed by calcimimetics: correlation with posttranslational modification of the trans acting factor AUF1. J. Am. Soc. Nephrol. 17:107-112.

4. Kilav, R., Silver, J., and Naveh-Many, T. 2001. A conserved cis-acting element in the parathyroid hormone 3 '-untranslated region is sufficient for regulation of RNA stability by calcium and phosphate. J. Biol.Chem. 276:8727-8733.

5. Kilav, R., Bell, O., Le, S.Y., Silver, J., and NavehMany, T. 2004. The parathyroid hormone mRNA 3 '-untranslated region AU-rich element is an unstructured functional element. J. Biol. Chem. 279:2109-2116.

6. Chen, C.Y., et al. 2001. AU binding proteins recruit the exosome to degrade ARE-containing mRNAs. Cell. 107:451-464.

7. Gherzi, R., et al. 2004. A KH domain RNA binding protein, KSRP, promotes ARE-directed mRNA turnover by recruiting the degradation machinery. Mol. Cell. 14:571-583.

8. Wilson, G.M., and Brewer, G. 1999. Identification and characterization of proteins binding $\mathrm{A}+\mathrm{U}$-rich elements. Methods. 17:74-83.

9. Wilusz, C.J., and Wilusz, J. 2004. Bringing the role of mRNA decay in the control of gene expression into focus. Trends Genet. 20:491-497.

10. Sela-Brown, A., Silver, J., Brewer, G., and Naveh- 
Many, T. 2000. Identification of AUF1 as a parathyroid hormone mRNA 3'-untranslated region binding protein that determines parathyroid hormone mRNA stability. J. Biol. Chem. 275:7424-7429.

11. Nechama, M., Ben Dov, I.Z., Briata, P., Gherzi, R., and Naveh-Many, T. 2008. The mRNA decay promoting factor K-homology splicing regulator protein post-transcriptionally determines parathyroid hormone mRNA levels. FASEB J. 22:3458-3468.

12. Nechama, M., Ben Dov, I.Z., Silver, J., and NavehMany, T. 2009. Regulation of PTH mRNA stability by the calcimimetic R568 and the phosphorus binder lanthanum carbonate in CKD. Am. J. Physiol. Renal Physiol. 296:F795-F800.

13. Wulf, G.M., Liou, Y.C., Ryo, A., Lee, S.W., and Lu, K.P. 2002. Role of Pin 1 in the regulation of p53 stability and p 21 transactivation, and cell cycle checkpoints in response to DNA damage. J. Biol. Chem. 277:47976-47979.

14. Zhou, X.Z., et al. 2000. Pin1-dependent prolyl isomerization regulates dephosphorylation of $\mathrm{Cdc} 25 \mathrm{C}$ and tau proteins. Mol. Cell. 6:873-883.

15. Lu, K.P., and Zhou, X.Z. 2007. The prolyl isomerase PIN1: a pivotal new twist in phosphorylation signalling and disease. Nat. Rev. Mol. Cell Biol. 8:904-916

16. Yaffe, M.B., et al. 1997. Sequence-specific and phosphorylation-dependent proline isomerization: a potential mitotic regulatory mechanism. Science. 278:1957-1960.

17. Wulf, G., Finn, G., Suizu, F., and Lu, K.P. 2005 Phosphorylation-specific prolyl isomerization: is there an underlying theme? Nat. Cell Biol. 7:435-441.

18. Winkler, K.E., Swenson, K.I., Kornbluth, S., and Means, A.R. 2000. Requirement of the prolyl isomerase Pin1 for the replication checkpoint. Science. 287:1644-1647.

19. Lu, P.J., Wulf, G., Zhou, X.Z., Davies, P., and Lu, K.P. 1999. The prolyl isomerase Pin 1 restores the function of Alzheimer-associated phosphorylated tau protein. Nature. 399:784-788.

20. Shen, Z.J., Esnault, S., and Malter, J.S. 2005. The peptidyl-prolyl isomerase Pin 1 regulates the stability of granulocyte-macrophage colony-stimulating factor mRNA in activated eosinophils. Nat. Immunol. 6:1280-1287.

21. Shen, Z.J., et al. 2008. Pin 1 regulates TGF-beta1 production by activated human and murine eosinophils and contributes to allergic lung fibrosis. J. Clin. Invest. 118:479-490.

22. Naveh-Many, T., and Nechama, M. 2007. Regulation of parathyroid hormone mRNA stability by calcium, phosphate and uremia. Curr.Opin. Nephrol. Hypertens. 16:305-310.

23. Hennig, L., et al. 1998. Selective inactivation of parvulin-like peptidyl-prolyl cis/trans isomerases by juglone. Biochemistry. 37:5953-5960.

24. Ben Dov, I.Z., et al. 2007. The parathyroid is a target organ for FGF23 in rats. J. Clin. Invest. 117:4003-4008.

25. Ranganathan, R., Lu, K.P., Hunter, T., and Noel, J.P. 1997. Structural and functional analysis of the mitotic rotamase Pin 1 suggests substrate recognition is phosphorylation dependent. Cell. 89:875-886.

26. Briata, P., et al. 2005. p38-dependent phosphorylation of the mRNA decay-promoting factor KSRP controls the stability of select myogenic transcripts. Mol. Cell. 20:891-903.

27. Gherzi, R., et al. 2006. The RNA-binding protein KSRP promotes decay of beta-catenin $\mathrm{MRNA}$ and is inactivated by PI3K-AKT signaling. PLoS Biol. 5:e5.

28. Fujimori, F., Takahashi, K., Uchida, C., and Uchida, T. 1999. Mice lacking Pin 1 develop normally, but are defective in entering cell cycle from $\mathrm{G}(0)$ arrest. Biochem. Biophys. Res. Commun. 265:658-663.

29. Wilson, G.M., et al. 2003. Phosphorylation of p40AUF1 regulates binding to A + U-rich mRNAdestabilizing elements and protein-induced changes in ribonucleoprotein structure. J. Biol. Chem.
278:33039-33048.

30. Fila, C., Metz, C., and van der Sluijs, P. 2008. Juglone inactivates cysteine-rich proteins required for progression through mitosis. J. Biol. Chem. 283:21714-21724.

31. Kifor, O., et al. 2001. Regulation of MAP kinase by calcium-sensing receptor in bovine parathyroid and CaR-transfected HEK293 cells. Am. J. Physiol. Renal Pbysiol. 280:F291-F302.

32. Galitzer, H., et al. 2009. The calcium-sensing receptor regulates parathyroid hormone gene expression in transfected HEK293 cells. BMC Biol. 7:17.

33. Lee, T.H., et al. 2009. Essential role of Pin 1 in the regulation of TRF1 stability and telomere maintenance. Nat. Cell Biol. 11:97-105.

34. Pani, E., et al. 2008. Pin 1 interacts with c-Myb in a phosphorylation-dependent manner and regulates its transactivation activity. Biochim. Biophys. Acta. 1783:1121-1128.

35. Ignatovich, I.A., et al. 2003. Complexes of plasmid DNA with basic domain 47-57 of the HIV-1 Tat protein are transferred to mammalian cells by endocytosis-mediated pathways. J. Biol. Chem. 278:42625-42636.

36. Wagner, B.J., DeMaria, C.T., Sun, Y., Wilson, G.M., and Brewer, G. 1998. Structure and genomic organization of the human AUF1 gene: alternative premRNA splicing generates four protein isoforms. Genomics. 48:195-202.

37. DeMaria, C.T., and Brewer, G. 1996. AUF1 binding affinity to $\mathrm{A}+\mathrm{U}$-rich elements correlates with rapid mRNA degradation. J. Biol. Chem. 271:12179-12184.

38. Lu, P.J., Zhou, X.Z., Liou, Y.C., Noel, J.P., and Lu, K.P. 2002. Critical Role of WW Domain Phosphorylation in Regulating Phosphoserine Binding Activity and Pin1 Function. J. Biol. Chem. 277:2381-2384.

39. Kilav, R., Silver, J., and Naveh-Many, T. 1995. Parathyroid hormone gene expression in hypophosphatemic rats. J. Clin. Invest. 96:327-333. 